\title{
EXISTENCE OF POSITIVE SOLUTIONS OF QUASILINEAR ELLIPTIC EQUATIONS
}

\author{
Adrian Constantin
}

We prove under quite general assumptions the existence of a positive solution to the equation $\Delta u+f(x, u)+g(x) x \cdot \nabla u=0$ in exterior domains of $R^{n}(n \geqslant 3)$.

Let us consider the quasilinear second order elliptic equation

$$
\Delta u+f(x, u)+g(|x|) x . \nabla u=0, \quad x \in G_{A},
$$

in an exterior domain $G_{A}=\left\{x \in R^{n}:|x|>A\right\}$. (Here $n \geqslant 3$ and $A>0$.)

Our purpose is to prove under quite general assumptions on the functions $f$ and $g$ the existence of a positive solution to (1) in $G_{B}=\left\{x \in R^{n}:|x|>B\right\}$ for some $B \geqslant A$.

Given that all solutions of (1) that are radial functions (which depend only on $r=|x|$ ) satisfy a second-order nonlinear ordinary differential equation, it seems natural to consider first the problem of the existence of solutions of constant sign to second order nonlinear ordinary differential equations. This ODE approach will enable us to construct a positive subsolution $w$ and a positive supersolution $v$ to (1) such that $w(x) \leqslant v(x), x \in G_{B}$, for some $B \geqslant A$, and then to establish the existence of a positive solution of $(1)$ in $G_{B}$ that is squeezed between $w(x)$ and $v(x)$.

Among the equations of the form (1) we have the equation

$$
\Delta u+f(x, u)=0, \quad x \in G_{A} .
$$

To show the applicability and usefulness of our results we show that they cover cases when recent investigations (see [4]) on equation (2) are powerless.

Received 27th September, 1995.

Copyright Clearance Centre, Inc. Serial-fee code: 0004-9729/96 \$A2.00+0.00. 
2.

Let us consider the second order elliptic equation

$$
\Delta u+F(x, u, \nabla u)=0, \quad x \in G_{A} \subset R^{n}
$$

subject to the assumptions

(i) there is a number $\alpha \in(0,1)$ such that $F \in C^{\alpha}(\bar{M} \times \bar{J} \times \bar{N}, R)$ (Hölder continuous) for every bounded domain $M \subset G_{A}$, every bounded interval $J \subset R$ and every bounded domain $N \subset R^{n}$;

(ii) for every bounded subdomain $M$ of $G_{A}$, there exists a nonnegative continuous function $l_{M}$ such that

$$
|F(x, t, y)| \leqslant l_{M}(|t|)\left(1+|y|^{2}\right), \quad x \in \bar{M}, t \in R, y \in R^{n}
$$

A solution $u$ of (3) in $G_{B}$ for some $B \geqslant A$ is defined to be a function $u \in$ $C^{2+\alpha}(\bar{M})$ for every bounded subdomain $M \subset G_{B}$, such that $u$ satisfies (3) at every point $x \in G_{B}$. Subsolutions of (3), that is, functions $u$ satisfying $\Delta u+F(x, u, \nabla u) \geqslant 0$, and supersolutions, that is, functions $u$ satisfying $\Delta u+F(x, u, \nabla u) \leqslant 0$, are defined similarly.

Let us denote for $B \geqslant A$,

$$
S_{B}=\left\{x \in R^{n}:|x|=B\right\}
$$

We shall need the following result in the sequel.

Lemma 1. [3] Assume that the conditions (i) and (ii) are satisfied. If for some $B \geqslant A$ there exists a positive subsolution $w$ and a positive supersolution $v$ to (3) in $G_{B}$ such that $w(x) \leqslant v(x)$ for all $x \in G_{B} \cup S_{B}$, then (3) has a solution $u$ in $G_{B}$ such that $w(x) \leqslant u(x) \leqslant v(x)$ throughout $G_{B} \cup S_{B}$ and $u(x)=v(x)$ for $x \in S_{B}$.

3.

Let us consider the nonlinear ordinary differential equation

$$
u^{\prime \prime}+G\left(t, u, u^{\prime}\right)=0, \quad t \geqslant 1
$$

where $G \in C^{1}\left([1, \infty) \times R^{2}, R\right)$.

We introduce the class $\Re$ of functions $w \in C^{1}\left(R_{+}, R_{+}\right)$with $w(0)=0$ and $w(t)>$ 0 for $t>0$, nondecreasing on $R_{+}$and which satisfy $\int_{1}^{\infty}(1 / w(s)) d s=\infty$. 


\section{LEMMA 2. Assume that}

$$
|G(t, u, v)| \leqslant a(t) w\left(\frac{|u|}{t}\right)+b(t)|v|, \quad t \geqslant 1, u, v \in R
$$

where $w \in \Re$ and $a, b \in C\left(R_{+}, R_{+}\right)$satisfy $\int_{0}^{\infty}[a(s)+b(s)] d s<\infty$.

Then equation (4) has a solution $u(t)$ which is of constant sign in $[m, \infty)$ for some $m \geqslant 1$.

Proof: Under the hypotheses of Lemma 2 we know (see [1]) that every solution of (4) is defined on $[1, \infty)$ and that for every solution $u(t)$ of $(4)$ there are real constants $c, d$ such that $u(t)=c t+d+o(t)$ as $t \rightarrow \infty$.

We shall actually show that any nontrivial solution of (4) is of constant sign in some interval $[m, \infty)$ for some $m \geqslant 1$.

Assume that there is a nontrivial solution $u(t)$ of $(4)$ which has a strictly increasing sequence of zeros $\left\{t_{n}\right\}_{n \geqslant 1}$ accumulating at $\infty$. Then we have that the corresponding $c, d$ are both equal to zero and taking into account (see [1]) that $c=\lim _{t \rightarrow \infty} u^{\prime}(t)$, this implies that $\lim _{t \rightarrow \infty} u^{\prime}(t)=\lim _{t \rightarrow \infty} u(t)=0$.

Since $u(t)$ is bounded on $[1, \infty)$, we may define

$$
K=\sup _{t \geqslant 1}\{|u(t)|\}, \quad L=\sup _{|u| \leqslant K}\left\{\left|w^{\prime}(u)\right|\right\}
$$

By the mean-value theorem (since $w(0)=0$ ) we have that $w(|u|) \leqslant L|u|$ for $|u| \leqslant K$.

Let $t_{k}>1$ be a root of $u(t)$ such that $\int_{t_{k}}^{\infty}[a(s)+b(s)] d s<1 /(L+1)$. Since $G$ is of class $C^{1}$ we have uniqueness for the solutions of (4) and so, since $u\left(t_{k}\right)=0$ and $u(t)$ is nontrivial, we have that $\left|u^{\prime}\left(t_{k}\right)\right|>0$ (otherwise $u(t)=0$ for all $t \geqslant 1$ since $G(t, 0,0)=0$ for all $t \geqslant 1)$. Since $\lim _{t \rightarrow \infty} u^{\prime}(t)=0$, there is a root $t_{n}>t_{k}$ of $u(t)$ with $\left|u^{\prime}(t)\right|<\left|u^{\prime}\left(t_{k}\right)\right| / 2$ for $t \geqslant t_{n}$. If $T \in\left[t_{k}, t_{n}\right]$ is a point where $\left|u^{\prime}(t)\right|$ attains its maximum on this interval, by the previous construction we have that

$$
\left|u^{\prime}(t)\right| \leqslant\left|u^{\prime}(T)\right|, \quad t \geqslant t_{k},
$$

and since $\left|u^{\prime}\left(t_{k}\right)\right|>0$ we also have that $\left|u^{\prime}(T)\right|>0$.

In view of the relation $T \geqslant t_{k}$ we get by the mean-value theorem that

$$
|u(s)|=\left|u(s)-u\left(t_{k}\right)\right| \leqslant\left(s-t_{k}\right)\left|u^{\prime}(T)\right|, \quad t \geqslant T,
$$

so that

$$
\frac{|u(s)|}{s} \leqslant\left|u^{\prime}(T)\right|, \quad s \geqslant T
$$


By integrating (4) on $[T, t]$, we get

$$
u^{\prime}(t)-u^{\prime}(T)+\int_{T}^{t} G\left(s, u(s), u^{\prime}(s)\right) d s=0, \quad t \geqslant T
$$

thus

$$
\left|u^{\prime}(T)\right| \leqslant\left|u^{\prime}(t)\right|+\int_{T}^{t} a(s) w\left(\frac{|u(s)|}{s}\right) d s+\int_{T}^{t} b(s)\left|u^{\prime}(s)\right| d s, \quad t \geqslant T .
$$

Since $\lim _{t \rightarrow \infty} u^{\prime}(t)=0$, we obtain taking into account the previous remarks that

$$
\begin{aligned}
\left|u^{\prime}(T)\right| & \leqslant \int_{T}^{\infty} a(s) w\left(\frac{|u(s)|}{s}\right) d s+\int_{T}^{\infty} b(s)\left|u^{\prime}(s)\right| d s \\
& \leqslant L\left|u^{\prime}(T)\right| \int_{T}^{\infty} a(s) d s+\left|u^{\prime}(T)\right| \int_{T}^{\infty} b(s) d s \\
& \leqslant\left|u^{\prime}(T)\right|(L+1) \int_{T}^{\infty}[a(s)+b(s)] d s<\left|u^{\prime}(T)\right|
\end{aligned}
$$

a contradiction.

This proves that any nontrivial solution $u(t)$ of $(4)$ is of constant sign in some interval $[m, \infty)$ for some $m \geqslant 1$.

4.

In order to be able to prove the main result of this paper, we recall from [2] the following useful fact

Lemma 3. [2] If $w \in \Re$, then the function defined on $R_{+}$by $t \rightarrow t+w(t)$ also belongs to the class $\Re$.

Theorem. Assume that there is a number $\alpha \in(0,1)$ such that $f \in C^{\alpha}(\bar{M} \times \bar{J}, R)$ for every bounded domain $M \subset G_{A}$ and every bounded interval $J \subset R$ and that $g \in C^{1}\left(R_{+}, R\right)$. If

$$
0 \leqslant f(x, t) \leqslant a(|x|) w(|t|), \quad t \in R_{+}, x \in R^{n}
$$

where $w \in \Re$ and $a \in C\left(R_{+}, R_{+}\right)$, then there is a positive solution to (1) on $G_{B}$ for some $B \geqslant A$ if

$$
\int_{0}^{\infty} s[a(s)+|g(s)|] d s<\infty
$$

Proof: Let us consider the differential equation

$$
\frac{d}{d r}\left\{r^{n-1} \frac{d y}{d r}\right\}+r^{n-1} a(r) w_{0}(y)+r^{n} g(r) \frac{d y}{d r}=0, \quad r \geqslant 1,
$$


where $w_{0}(t)=w(t)$ for $t \in R_{+}$and $w(t)=-w(|t|)$ for $t \leqslant 0$ (since $w(0)=0$ we see that $\left.\boldsymbol{w}_{0} \in C^{1}\left(R_{+}, R_{+}\right)\right)$.

The change of variables

$$
r=\beta(s)=\left\{\frac{1}{n-2} s\right\}^{1 /(n-2)}, \quad h(s)=s y(\beta(s)),
$$

transforms (5) into

$$
\begin{aligned}
h^{\prime \prime}(s) & +\frac{1}{n-2} \beta^{\prime}(s) \beta(s) a(\beta(s)) w_{0}\left(\frac{h(s)}{s}\right) \\
& +\beta^{\prime}(s) \beta(s) g(\beta(s))\left\{h^{\prime}(s)-\frac{h(s)}{s}\right\}=0 .
\end{aligned}
$$

In view of Lemma 3 we have that the function $t \rightarrow t+w(t), t \in R_{+}$, belongs to $\Re$, so that, since

$$
\int_{0}^{\infty}\left\{\beta^{\prime}(s) \beta(s) a(\beta(s))+\beta^{\prime}(s) \beta(s)|g(\beta(s))|\right\} d s=\int_{0}^{\infty} s[a(s)+|g(s)|] d s<\infty,
$$

we deduce by Lemma 2 that there is a solution to (6) which has a constant sign on some interval $[m, \infty)$ with $B=\beta(m) \geqslant A$.

Returning to (5), this yields a solution of (5) which has a constant sign on some interval $[B, \infty)$ with $B \geqslant A$. Since $w_{0}$ is odd on $R$, we observe that if $y(r)$ is a solution of (5), then $-y(r)$ is also a solution; thus we can state that (5) has a positive solution on some interval $[B, \infty)$ with $B \geqslant A$.

Let us define $v(x)=y(r), r=|x| \geqslant B$.

We have that $v(x)>0$ on $S_{B} \cup G_{B}$ and

$$
\begin{aligned}
r^{n-1} \Delta v(x) & =\frac{d}{d r}\left\{r^{n-1} \frac{d y}{d r}\right\}+r^{n-1} f(x, v(x))+r^{n-1} g(r) x . \nabla v(x) \\
& =\frac{d}{d r}\left\{r^{n-1} \frac{d y}{d r}\right\}+r^{n-1} f(x, v(x))+r^{n} g(r) \frac{d y}{d r} \\
& \leqslant \frac{d}{d r}\left\{r^{n-1} \frac{d y}{d r}\right\}+r^{n-1} a(r) w(y(r))+r^{n} g(r) \frac{d y}{d r}=0, \quad r \geqslant B,
\end{aligned}
$$

so that $v$ is a supersolution to (1) on $G_{B}$. Clearly $w(x)=0$ satisfies

$$
\Delta w(x)+f(x, w(x))+g(|x|) x . \nabla w(x) \geqslant 0, \quad x \in G_{B} .
$$

By Lemma 1 we deduce that (1) has a solution $u(x)$ in $G_{B}$ with $w(x) \leqslant u(x) \leqslant$ $v(x)$ for $|x|>B$ and $u(x)=v(x)$ for $|x|=B$. 
Since $\int_{0}^{\infty} s|g(s)| d s<\infty$ there is $k>0$ such that

$$
\sup _{t \geqslant 1}\{|g(t)|\}+\frac{n}{B^{2}} \leqslant k
$$

Define for $\varepsilon>0$,

$$
u_{\varepsilon}(x)=\inf _{z \in S_{B}}\{u(z)\}+\varepsilon e^{-k|x|^{2}}, \quad x \in S_{B} \cup G_{B},
$$

where $u(x)$ is the solution of (1) in $G_{B}$, and let us consider the operator

$$
\left.L z=\Delta z+g(|x|) x . \nabla z, \quad z \in C^{2}\left(G_{B}\right) \cap C{\left(G_{B}\right.}\right) .
$$

Observe that

$$
\begin{aligned}
L u_{\varepsilon} & =\varepsilon\left(4 k^{2}|x|^{2}-2 k n\right) e^{-k|x|^{2}}-2 \varepsilon k|x|^{2} g(|x|) e^{-k|x|^{2}} \\
& \geqslant 2 \varepsilon k^{2}|x|^{2} e^{-k|x|^{2}}-2 \varepsilon k|x|^{2} g(|x|) e^{-k|x|^{2}} \\
& \geqslant 2 \varepsilon k|x|^{2} e^{-k|x|^{2}}\left(k-\sup _{t \geqslant 0}\{|g(t)|\}\right)>0 \geqslant-f(x, u(x)) \\
& =\Delta u(x)+g(|x|) x \cdot \nabla u(x)=L\left(u+\varepsilon e^{-k B^{2}}\right), \quad x \in G_{B}
\end{aligned}
$$

that is,

$$
L\left(\left[u+\varepsilon e^{-k B^{2}}\right]-u_{\varepsilon}\right)<0, \quad x \in G_{B}
$$

On the other hand,

$$
\left[u(x)+\varepsilon e^{-k B^{2}}\right]-u_{\varepsilon}(x) \geqslant 0, \quad x \in S_{B}
$$

Since $u(x) \geqslant 0$ on $G_{B}$ and $u_{\varepsilon}(x)$ is bounded on $G_{B}$, we have that the function $z_{\varepsilon}(x)=u(x)+\varepsilon e^{-k B^{2}}-u_{\varepsilon}(x), x \in G_{B} \cup S_{B}$, has a finite infimum in $G_{B} \cup S_{B}$. If there was $x_{0} \in G_{B}$ with

$$
z_{\varepsilon}\left(x_{0}\right)=\inf _{x \in G_{B} \cup S_{B}}\left\{z_{\varepsilon}(x)\right\}
$$

we would have that $\Delta z_{\varepsilon}\left(x_{0}\right) \geqslant 0$ and $\nabla z_{\varepsilon}\left(x_{0}\right)=0$, so that $L z_{\varepsilon}\left(x_{0}\right) \geqslant 0$, which is not possible. Thus

$$
0 \leqslant \inf _{x \in S_{B}}\left\{z_{\varepsilon}(x)\right\}=\inf _{x \in G_{B} \cup S_{B}}\left\{z_{\varepsilon}(x)\right\}
$$

and we obtain

$$
u_{\varepsilon}(x) \leqslant u(x)+\varepsilon e^{-k|x|^{2}}, \quad x \in G_{B} \cup S_{B}
$$


By letting $\varepsilon \rightarrow 0$ in the previous relation, we get

$$
0<y(B)=\inf _{x \in S_{B}}\{u(x)\} \leqslant u(x), \quad x \in G_{B}
$$

so that $u(x)$ is positive in $G_{B}$.

As a Corollary of our theorem we have the following

Proposition. Assume that there is a number $\alpha \in(0,1)$ such that $f \in$ $C^{\alpha}(\bar{M} \times \bar{J}, R)$ for every bounded domain $M \subset G_{A}$ and every bounded interval $J \subset R$. If

$$
0 \leqslant f(x, t) \leqslant a(|x|) w(|t|), \quad t \in R, x \in R^{n},
$$

where $w \in \Re$ and $a \in C\left(R_{+}, R_{+}\right)$, then there is a positive solution to (2) on $G_{B}$ for some $B \geqslant A$ if

$$
\int_{0}^{\infty} s a(s) d s<\infty
$$

The following example shows the applicability of the proposition.

Example. Consider the quasilinear second order elliptic equation

$$
\Delta u+\frac{u \ln (1+|u|)}{1+|x|^{3}}=0, \quad x \in G_{1} \subset R^{3} .
$$

By our Proposition, there is a positive solution to (8) in some $G_{B}$ with $B \geqslant 1$. Observe that the results of Swanson [4] are not applicable.

Among the equations of the form (2) we have the sublinear Emden-Fowler equation

$$
\Delta u+p(x)|u|^{\gamma} \operatorname{sgn}(u)=0, \quad 0<\gamma<1, \quad x \in G_{A},
$$

where $p(x)$ is nonnegative and Hölder continuous in $G_{A}$ with $A>0$. An application of our proposition shows that if

$$
\int_{0}^{\infty} s \max _{|x|=s}\{p(x)\} d s<\infty
$$

then (9) has a positive solution in $G_{B}$ for some $B \geqslant A$. It is known (see [4]) that if

$$
\int_{0}^{\infty} s \min _{|x|=s}\{p(x)\} d s=\infty
$$

then all solutions of (9) are oscillatory. Thus if $p(x)=a(|x|)$, then the necessary and sufficient condition for the existence of a positive solution to (9) is condition (7) and this shows the sharpness of our results. 


\section{REFERENCES}

[1] A. Constantin, 'On the asymptotic behavior of second order nonlinear differential equations', Rend. Mat. Appl. 13 (1994), 627-634.

[2] A. Constantin, 'Solutions globales des équations différentielles perturbées,', C.R. Acad. Sci. Paris 320 (1995), 1319-1322.

[3] E.S. Noussair and C.A. Swanson, 'Positive solutions of quasilinear elliptic equations in exterior domains', J. Math. Anal. Appl. 75 (1980), 121-133.

[4] C.A. Swanson, 'Criteria for oscillatory sublinear Schrödinger equations', Pacific J. Math. 104 (1983), 483-493.

Courant Institute of Mathematical Sciences

251 Mercer Street

10012 New York NY

United States of America 